\title{
Norois
}

Environnement, aménagement, société

197 | 2005/4

Varia

\section{De nouveaux dirigeants de PMI pour le milieu rural. Enseignements d'étude de cas}

New SME managers for rural areas. The conclusions established from case studies

\section{Marie Raveyre}

\section{OpenEdition}

\section{Journals}

Édition électronique

URL : https://journals.openedition.org/norois/335

DOI : 10.4000/norois.335

ISBN : 978-2-7535-1545-1

ISSN : $1760-8546$

\section{Éditeur}

Presses universitaires de Rennes

\section{Édition imprimée}

Date de publication : 1 décembre 2005

Pagination : 83-94

ISBN : 978-2-7535-0238-3

ISSN : 0029-182X

\section{Référence électronique}

Marie Raveyre, " De nouveaux dirigeants de PMI pour le milieu rural. Enseignements d'étude de cas », Norois [En ligne], 197 | 2005/4, mis en ligne le 15 décembre 2008, consulté le 13 janvier 2022. URL :

http://journals.openedition.org/norois/335 ; DOI : https://doi.org/10.4000/norois.335

Ce document a été généré automatiquement le 13 janvier 2022.

(c) Tous droits réservés 


\title{
De nouveaux dirigeants de PMI pour le milieu rural. Enseignements d'étude de cas
}

\author{
New SME managers for rural areas. The conclusions established from case \\ studies
}

Marie Raveyre

\section{NOTE DE L'ÉDITEUR}

Cet article a été reçu le 9 juin 2005 et définitivement accepté le 28 novembre 2005.

1 L'avenir des entreprises industrielles en milieu rural paraît plus que jamais problématique. Nombres d'analyses ont mis en évidence une tendance au déclin, que le contexte de crise économique ne ferait qu'exacerber. Des entreprises ferment, des départements entiers sont marqués par la désertion d'activité industrielle. Ce constat, fondé sur des observations bien réelles, risque, en focalisant l'attention sur ce qui disparaît, de masquer l'émergence de nouvelles dynamiques de développement. La crise actuelle est aussi une recomposition du système productif: si des types de dynamiques industrielles entrent en désuétude, d'autres se mettent en place. Les atouts et handicaps de l'espace rural ne sont plus nécessairement du même ordre que par le passé, ceci d'autant plus que ce milieu a connu d'importantes évolutions sociales ces dernières décennies. Les entreprises changent et le monde rural aussi, leurs évolutions sont-elles fatalement de sens contraires? Pour contribuer à la réflexion sur ce thème nous avons choisi de focaliser l'attention sur des petites entreprises industrielles rurales en bonne santé, afin d'analyser les raisons de leur dynamisme et de dégager, ainsi, à titre prospectif, quelques pistes quant aux possibilités de développement des entreprises dans ce milieu. 
2 Les préconisations le plus souvent avancées pour soutenir l'industrialisation en milieu rural peuvent être regroupées schématiquement dans deux grandes perspectives. La première mise sur des facteurs exogènes, avec le recours à l'implantation d'établissements d'entreprises nées en ville, ou la réalisation d'activités de soustraitance pour ces dernières. On rejoint, ici, de fait, une conception liant industrialisation et urbanisation, dans laquelle le monde rural occupe une position périphérique. Dans ce cadre, si des entreprises se maintiennent c'est essentiellement parce qu'elles produisent pour des marchés de proximité, ou bien qu'elles prennent en charge certaines activités à moindre coût salarial. La seconde valorise des facteurs endogènes, avec l'incitation à la constitution de systèmes productifs locaux. En référence aux districts italiens (Bagnasco, 1977; Becattini, 1987) et aux ensembles industriels localisés centrés sur une spécialité, telle que la coutellerie à Thiers (Trouvé, 1989) ou la chaussure dans le Choletais (Courlet et Pecqueur, 1988), il s'agit d'encourager l'essor de réseaux d'entreprises complémentaires, notamment de PME, en misant sur des synergies de coopération entre les acteurs locaux. Au-delà de ces deux voies de développement, toujours d'actualité ${ }^{1}$, d'autres perspectives semblent pouvoir être dégagées pour l'industrialisation des espaces ruraux, et plus particulièrement en ce qui concerne les PME.

3 En effet, nos observations d'une vingtaine de PMI situées en milieu rural non périurbain, esquissent la possibilité d'une nouvelle voie. Les entreprises étudiées ne sont pas strictement nées du terroir, sans être pour autant des importations urbaines. Elles s'appuient sur des facteurs locaux, mais ne s'y limitent pas, car elles participent aussi à des dynamismes nationaux, voire internationaux; c'est précisément l'articulation de ces dimensions locales et globales qui ferait leur force.

4 En dépit de la variété de leur secteur d'activité et de la diversité de leur situation géographique, ces PMI, présentent des traits communs; ce qui nous conduit à les considérer comme appartenant à un même modèle socio-productif, caractérisé par un profil d'entrepreneur spécifique et un mode de fonctionnement adaptatif. Anciens cadres urbains, ces dirigeants, créateurs de leur activité, ont des comportements et des systèmes de valeurs éloignés de ceux des patrons traditionnels de PME. Le mode de fonctionnement de ces PMI porte la marque des représentations de leurs dirigeants en quête de nouveaux modes de management, envisageant sans réticence innovations technologiques et ouvertures commerciales. En effet, la performance de ces petites entreprises, que l'on ne peut qualifier de haute technologie, proviendrait de quatre orientations principales: la spécialisation sur des créneaux de marché, l'élasticité technique, l'intégration dans des réseaux interentreprises à la fois locaux et nationaux/ internationaux et la flexibilité du travail. Cet ensemble de traits communs esquisse les contours d'une forme particulière de PME, qui se démarque tant du modèle de la PME indépendante traditionnelle, proche de l'artisanat ${ }^{2}$, que de celui des systèmes productifs locaux.

5 Dans le cadre d'une approche socioéconomique, nous nous attacherons à analyser les particularités de ces PMI et la façon dont elles s'inscrivent dans le milieu rural, en s'interrogeant plus particulièrement quant aux facteurs ayant concouru à leur développement. Nous préciserons en premier lieu notre méthode et les caractéristiques de notre échantillon. Puis, à partir des lignes de forces qui se dégagent de celui-ci, nous aborderons successivement le profil des dirigeants, les stratégies de développement des entreprises et leur mode de gestion des ressources humaines. 


\section{Des PMI hors des chemins battus}

Les entreprises étudiées, sont créatrices d'emplois, - c'est suivant ce critère qu'elles ont été sélectionnées. Dispersées sur le territoire national en milieu rural non périurbain, outre leur bonne santé, elles présentent un profil relativement original de PMI indépendantes, créées aux cours des deux dernières décennies, ne se situant pas dans le secteur de l'agro-alimentaire. Si cette étude de cas ne saurait être représentative de l'état actuel des PME en milieu rural, elle est cependant significative du fait que des petites entreprises industrielles peuvent s'y développer.

\section{Des PMI en expansion en milieu rural non périurbain}

7 Les entreprises ont été observées de façon directe sur la base d'entretiens avec leurs dirigeants et de visites des locaux. L'étude de cas repose sur un échantillon de 21 PMI sélectionnées parmi une centaine de PME auprès desquelles nous avons mené des enquêtes, lors de plusieurs travaux de recherche portant sur des entreprises ayant bénéficié d'aides à la création d'emploi (Raveyre, 1988, 1999), en retenant de façon systématique les PME situées en milieu rural, hors des zones périurbaines. Il s'agit donc de l'exploitation secondaire de résultats d'enquêtes conduites lors d'études n'ayant pas pour problématique la question de l'industrialisation en zone rurale. L'une des particularités de cet échantillon est de ne retenir que des entreprises créatrices d'emploi, car c'est suivant ce critère que des aides aux entreprises ont été attribuées. Ce mode sélection permet ainsi de focaliser l'attention sur des PME en expansion.

8 Les PME étudiées sont réparties sur l'ensemble du territoire national, dans des communes éloignées des grands centres urbains (Attigny à $40 \mathrm{~km}$ de CharlevilleMézières, Aiton à $30 \mathrm{~km}$ d'Albertville, Mortagne-sur-Sèvre à $10 \mathrm{~km}$ de Cholet, Mauléon à $100 \mathrm{~km}$ de Lourdes, etc.). Quatre d'entre elles seulement sont établies dans de petites villes (Sens, Fontenay-le-Comte, Montbrison). Elles sont situées majoritairement dans des sites peu industrialisées, voire des sites où l'entreprise est la seule activité industrielle ${ }^{3}$. Elles n'appartiennent pas à des ensembles industriels locaux, même si l'une d'elles se situe dans ce type de territoire, car leur activité est généralement peu liée à celles des entreprises environnantes. Ainsi, l'entreprise du Choletais est une menuiserie, sans lien direct avec l'industrie de la chaussure locale.

\section{Des créations d'entreprises industrielles spécialisées}

9 Les entreprises ont été créées majoritairement au cours des deux dernières décennies : 18 d'entre elles ont été fondées par leur dirigeant actuel et 3 sont des reprises (tableau 1). Toutefois, ces reprises s'apparentent à des créations d'entreprise, car il s'agit de rachats de société en faillite qui ont été radicalement transformées : nouveaux bâtiments, changement de production. Par exemple, une entreprise de sous-traitance de pièces pour l'automobile, est devenue spécialiste mondiale de sonar, avec des bâtiments neufs et les trois quarts du personnel renouvelé.

On sait par ailleurs, qu'un mouvement de création d'établissements d'activité non agricole dans les zones rurales en France, a été identifié dès le début des années 1980, par plusieurs travaux statistiques, notamment ceux de J.-C. Bontron (1991). Dans le 
même sens, P.-A. Julien et M. Marchesnay (1988), soulignent que, les PME manufacturières de moins de cent salariés sont désormais en proportion plus importante dans certaines zones, notamment du sud de la France, «là où l'industrie avait pas ou peu de passé ». Notre étude de cas s'inscrirait donc dans un mouvement plus général de regain de création d'entreprise hors des bassins industriels existants et des pôles urbains.

\begin{tabular}{|l|c|c|c|}
\hline & Date de fondation & Total \\
\hline Type d'entreprise & $1968-1979$ & $1980-1995$ & 18 \\
\hline Création & 7 & 11 & 3 \\
Reprise & 1 & 2 & 21 \\
\hline Total & 8 & 13 & \\
\hline
\end{tabular}

Tableau 1 : Répartition des PME par type d'entreprise et date de création Distribution of SMEs according to type of business and date of establishment

11 Les PME ont une moyenne de 35 salariés et appartiennent à différents secteurs d'activité (tableau 2). Une seule d'entre elles appartient au secteur agroalimentaire avec la fabrication de biscuits ; une relève du secteur des services, avec des activités de recherche-développement de procédés pour l'industrie. Les autres se situent majoritairement dans la production de biens intermédiaires (pièces et ensembles techniques, emballages spéciaux, transformation de matériaux, pour diverses industries). On trouve quatre cas de production de biens de consommation finale: horlogerie, automobile (fabrication de 4x4), menuiserie, biscuiterie.

\begin{tabular}{|l|c|c|c|c|c|}
\hline \multicolumn{1}{|c|}{ Secteur } & \multicolumn{5}{|c|}{ Effectif } \\
\hline \multicolumn{1}{|c|}{} & 10 & $0-25$ & $6-50$ & $50-200$ & Total \\
\hline Mécanique & 2 & 1 & 3 & 2 & 8 \\
Électronique \& informatique & 1 & 1 & 1 & 1 & 4 \\
Transformation de matières plastiques & 1 & 2 & 1 & & 4 \\
Transformation de matériaux spéciaux & & 1 & 2 & & 3 \\
Industrie du bois & & 2 & & & 2 \\
\hline Total & 4 & 7 & 7 & 3 & 21 \\
\hline
\end{tabular}

Tableau 2 : Répartition des PME par secteur d'activité et par effectif salarié Distribution of SMEs according to sector of activity and workforce

12 Aucune des entreprises ne se trouve en situation de sous-traitant classique (tableau 3), c'est-à-dire assurant des fonctions d'exécution pour des donneurs d'ordres. Celles qui sont sous-traitantes le sont de spécialité, gardant la maîtrise de la conception des produits. Elles sont donc en position relativement indépendantes, ce qui est d'autant plus le cas de la majorité des entreprises de l'échantillon, qui réalisent des produits propres.

\begin{tabular}{|c|c|}
\hline Sous-traitant de spécialité & Produits propres \\
\hline 6 & 15 \\
\hline
\end{tabular}

Tableau 3 : Répartition des PME suivant leur type de fabrication

Distribution of SMES according to their type of production

13 Ainsi les PME étudiées présentent des caractéristiques communes particulièrement marquées, malgré la diversité de leur secteur d'activité et de leur localisation. Cette convergence est encore plus manifeste à l'examen des spécificités socio-productives des entreprises. 


\section{Des néo-entrepreneurs ruraux} contrairement à ceux que l'on prête souvent au milieu rural, particulièrement quand il s'agit de création d'activité. Hors du schéma classique, ils le sont aussi relativement au modèle du patron de PME traditionnel. En référence au modèle du cercle familial élargi (Bunel et Saglio, 1979), celui-ci peut être défini comme correspondant à un pouvoir managérial paternaliste et à des valeurs d'ordre familial où, notamment, la possession du capital est considérée comme un bien patrimoine transmis de génération en génération.

\section{Des cadres diplômés quittant la ville}

15 Majoritairement d'origine urbaine, ces dirigeants, pour la moitié d'entre eux, ne sont pas nés dans la région d'implantation de leur entreprise. La filiation au milieu rural est donc faible. S'il est vrai que pour certains il y a actuellement identité entre lieu de naissance et lieu de travail, c'est au sens large d'une région, et non pas d'une commune. Dans leur ensemble, le parcours de ces entrepreneurs montre une grande mobilité géographique, nombre d'entre eux ont vécu dans d'autres régions, parfois même à l'étranger.

16 L'image classique de chef d'entreprise d'aussi petite taille, renvoie à un profil de personne de niveau de diplôme peu élevé, ayant assuré auparavant des fonctions de chef d'atelier ou de technicien. Or ce cas de figure est ici fort rare : nous sommes en présence essentiellement de cadres : $86 \%$ d'entre eux ont un niveau supérieur au BAC, dont $61 \%$ ayant une formation supérieure à BAC+3. Si l'augmentation du niveau de formation des créateurs d'entreprise depuis le milieu des années soixante-dix, a été souligné (Guyot, 1987) elle apparaît cependant particulièrement conséquente dans les cas que nous avons observés. Pour prendre un point de comparaison, l'enquête SINE de l'INSEE (1996), montre que les créateurs et repreneurs d'entreprise, sur l'ensemble du territoire national, en 1994, ont une formation supérieure au BAC pour 40,6\% d'entre eux, dont $13,2 \%$ avec une formation supérieure à $\mathrm{BAC}+3^{4}$.

17 Le profil de dirigeant qui se dégage ici paraît donc très spécifique, il semblerait significatif d'un certain changement social. En effet, ces dirigeants, de formation supérieure (voire des plus prestigieuses, telles que l'ENA et polytechnique), abandonnant des fonctions de cadre pour fonder leur entreprise aux alentours de la quarantaine, ne semblaient pas tenus d'adopter une position de retrait ${ }^{5}$. Aussi, ce mouvement de retour à la PME serait significatif d'une modification de système de valeurs, comme semblent le confirmer les discours de ces dirigeants. L'analyse de ceuxci, fait apparaître une représentation particulière de l'entreprise, dans laquelle la grande entreprise n'est pas le modèle idéal de référence et la petite celui de la médiocrité. Au contraire, la PME est présentée comme un lieu privilégié d'exercice effectif du pouvoir managérial, opposée aux lourdeurs de structure, et à la sclérose des grandes entreprises. En cela, ces dirigeants ne sont pas étrangers au mouvement de revalorisation de la PME des années 1980, dont une certaine presse managériale s'est faite l'écho.

Norois, 197 | 2005/4 

de retour aux origines, bien que celles-ci soient plus électives que réelles, semble lié à un goût pour l'environnement naturel. Souvent, ce dernier a été déterminant dans le choix du lieu d'implantation, les sites étant jugés relativement équivalents du point de vue des ressources productives et des systèmes d'aides locaux, qui sont fréquemment présents dans ces zones rurales. Cet attrait pour la nature se retrouve dans l'aménagement des locaux, où un souci de mise en valeur de l'environnement existe : une entreprise est installée dans une vieille ferme restaurée dans le style rustique, une autre a un atelier avec une verrière donnant sur la forêt, etc. On remarquera que cet intérêt pour la nature paraît plus correspondre à un système de valeur propre à une certaine catégorie de cadres urbains qu'à celui de patrons traditionnels de PME rurales.

\section{Des dirigeants, pas des patrons}

Si ces dirigeants se montrent critiques à l'égard des grandes entreprises, ils ne reconduisent cependant pas le modèle de la PME familiale traditionnelle. Leur entreprise n'apparaît pas fortement articulée autour de la parenté : la famille travaille dans l'entreprise que dans un tiers des cas, et seulement un quart des épouses de dirigeants participent à l'activité. Celles-ci exercent souvent une activité de qualification supérieure : enseignement, médecine, etc. Par ailleurs, la très grande majorité de ces dirigeants n'envisagent pas que leurs enfants prennent leur succession. Enfin, ces entrepreneurs considèrent leur entreprise plutôt comme un outil que comme un bien patrimonial. Si pour eux la possession du capital est toujours une garantie d'autonomie, cette propriété est souvent partagée: dans les deux tiers des cas, les entreprises observées présentent une direction collégiale. Les associés, de compétences complémentaires, sont souvent des cofondateurs qui se sont choisis selon des critères affinitaires, au sein de réseaux de collègues de travail ou d'anciens élèves. En s'associant pour créer une entreprise, ces dirigeants font partie d'un phénomène, relativement nouveau, qui a pu être observé ailleurs ${ }^{6}$.

Ces chefs d'entreprise ne reproduisent donc guère le modèle de la PME familiale. Ils récusent d'ailleurs le vocable de patron qu'ils rattachent à cette dernière. Se désignant comme des dirigeants, ils invoquent un autre type de légitimité. Critiques autant de la rigidité bureaucratique de la grande entreprise classique, que du traditionalisme de la PME familiale, ces dirigeants présentent leur entreprise comme une équipe. Cette représentation de l'entreprise, semble correspondre aux particularités culturelles de ce sous-groupe social de cadres. Ces derniers valorisent l'autonomie et le retour à des principes plus conviviaux, tout en récusant l'individualisme. Leur idéal d'entreprise se présente comme un modèle d'entreprise à taille humaine, dont la réussite ne peut être que collective. On remarquera que cette conception de l'organisation de l'entreprise s'apparente aux nouveaux modes de management participatif, donnant une place centrale à la mobilisation des ressources humaines.

21 Pour que ce modèle d'adhésion perdure, la grande majorité de ces dirigeants n'envisage pas que l'entreprise dépasse un effectif de cinquante salariés. Dès lors, le développement de l'activité doit, pour eux, se faire en créant des sociétés satellites, des quasi-filiales autonomes, donnant parfois naissance à des micro-groupes ${ }^{7}$. Ce principe a déjà été mis en pratique par le tiers d'entre eux. Ce souci de maintenir de petites unités correspond aussi à la recherche d'une plus grande souplesse productive. L'entreprise 
doit pouvoir s'adapter, et pour cela, éviter centralisme et concentration, afin d'exploiter à moindre risque des créneaux de marché, qui lorsqu'ils disparaissent n'affectent alors qu'un seul satellite.

La tendance d'ensemble dessine donc un profil de créateur d'entreprise relativement original, de cadres retournant à la campagne et à la PME, des néo-entrepreneurs ruraux, dont les caractéristiques ne sont pas à rattacher à un milieu local spécifique, mais à une catégorie de cadres en quête d'autres modes de vie et d'autres façons d'exercer leur activité professionnelle. Le milieu rural ne serait donc pas déserté par les nouvelles formes d'entreprenariat. La représentation de l'entreprise de ces dirigeants a joué un rôle moteur dans les orientations qui ont été données à ces entreprises.

\section{Des PMI adaptatives}

Si les PME étudiées sont en expansion, la raison de leur performance ne se trouve pas du côté de la mise en œuvre de véritables innovations de produits ou de procédés. Elle paraît résider en une logique particulière, mise en œuvre dès leur création. Misant sur la qualité du produit, la stratégie commerciale de ces PME s'articule étroitement avec une valorisation ingénieuse des ressources technologiques et une mobilisation de réseaux interentreprises dépassant le niveau local.

\section{La carte de la spécialisation}

Pour définir leurs marchés, les dirigeants ont opté pour des stratégies qui pouvaient leur assurer le plus possible d'autonomie et de compétitivité. Ils ont cherché à éviter les situations de sous-traitance captive, jugeant la dépendance avec le donneur d'ordres trop contraignante. Comme nous l'avons évoqué, très majoritairement, les PME étudiées ont des produits propres, ou assurent de la sous-traitance de spécialité. Elles maitrisent donc la conception et le savoir-faire de fabrication. D'autre part, ces entrepreneurs ont misé sur des produits à forte valeur ajoutée, laissant ainsi de côté les produits de consommation de masse, qu'ils considèrent trop fortement concurrencés et nécessitant des équipements lourds et rigides. Leur stratégie concurrentielle consiste à jouer la carte de la spécialisation, en misant sur la qualité du produit, plutôt que sur le faible prix, ainsi que sur l'adaptation à la demande du client. En cela, elle se trouve en phase avec l'évolution générale des critères de la concurrence internationale, tels qu'ils s'affirment depuis les années 1980, dans lesquels la qualité, la variété, la réactivité et l'innovation occupent désormais une place importante (Veltz, 2002).

L'originalité de leurs produits ne frappe pas un œil non averti, car elle repose fréquemment sur des astuces exclusives et des adaptations inédites: ainsi l'une des PME fabrique des pavés en verre dont la forme permet un assemblage jusque-là inégalé, une autre est la spécialiste mondiale de la pompe hydraulique silencieuse, une troisième de matériaux "à mémoire de forme ", etc. Spécialistes, ces PME le sont ainsi du mouton à cinq pattes, suivant les termes de leurs dirigeants. Sur la base de microinnovations de produits, ces entreprises se différencient des autres sur le marché. Par cette politique de produit, ces PME s'éloignent des relations de dépendance, car elles se positionnent, de fait, en concepteurs de produits. Dans cette stratégie, il s'agit de valoriser des créneaux dans les interstices laissés vacants par les concurrents; créneaux qui ne sont pas limités à un marché local, mais qui leur assurent une 
ouverture nationale, et, pour plusieurs d'entre elles, internationale. On remarquera que ces anciens cadres, contrairement à certains patrons de PME traditionnelles, envisagent sans réticence toutes les possibilités de développement commercial.

Une telle stratégie de petits créneaux spécialisés, nécessite, pour être durable, que l'entreprise soit très mobile sur le marché, afin de se déplacer suivant les opportunités, adaptant sans cesse produits et procédés, (ceci à moindre frais d'investissement). Aussi, cette stratégie apparaitt-elle liée au mode de mise en œuvre des techniques particulier à ces PME, reposant sur la flexibilité.

\section{Une valorisation ingénieuse des techniques}

Le souci d'éviter la rigidité a été souvent l'un des critères directeurs lors du choix des équipements productifs. Ouverts aux nouvelles technologies, ces anciens cadres y recourent de façon modérée (de l'ordre de $50 \%$ du parc machines). L'observation des procédés de fabrication fait alors apparaître une curieuse coexistence de celles-ci avec des techniques plus anciennes. Par exemple, on trouve dans le même atelier, un système de conception assisté par ordinateur (CAO) et un tour mécanique classique ou des soudeuses manuelles. Si pareil éclectisme peut surprendre, il n'apparaît pas sans rationalité. Outre le fait que ceci est moins onéreux, ces deux techniques de générations différentes ont pour caractère commun d'être modulaire ; c'est-à-dire que chaque machine se présente comme une unité ne dépendant pas nécessairement d'un ensemble, contrairement à celles de la génération intermédiaire (automatisme simple de type ligne de montage). Ce type d'équipement présente l'avantage de pouvoir fractionner les investissements suivant les capitaux disponibles, puisque chaque machine peut être achetée à l'unité. Par ailleurs, cette plus grande autonomie des techniques explique que ces petites entreprises du milieu rural puissent fonctionner en l'absence de proche environnement industriel, du fait de leur moindre dépendance de procès.

L'association de ces techniques, si elle peut paraître peu orthodoxe, n'en donne pas moins lieu à des bricolages inédits, à des innovations fort discrètes, qui constituent une partie du capital de secrets de fabrication de l'entreprise. Mais aussi, le recours à ces équipements permet de mener à bien la stratégie du créneau de marché. En effet, les techniques utilisées ont pour caractéristiques de permettre des fabrications de haut niveau et flexibles (permettant de changer de produit sans changer d'équipement et de fabriquer tant à l'unité qu'en petites séries). C'est en valorisant ces possibilités que ces PME proposent des produits spécialisés pouvant être modifiés suivant la demande des clients.

On notera, que si certains avaient fondé des espoirs dans les nouvelles technologies de communication, qui devaient permettre le développement du télétravail en milieu rural, il semble que l'on ait sous-estimé les possibilités de revitalisation des PME de ce milieu par l'utilisation des nouvelles technologies de production. Ces dernières plus autonomes et flexibles sont intégrables dans de très petites entreprises, qui peuvent ainsi atteindre un haut niveau de performance, même si elles sont isolées sur le territoire. Le rôle des nouvelles technologies de communication est cependant important pour l'essor de ces entreprises, de même que le développement des infrastructures de transport : tous deux opèrent un certain désenclavement de l'espace rural, facilitant l'accès tant aux informations qu'aux marchés. 


\section{Une articulation de réseaux locaux et globaux}

Les réseaux interentreprises dans lesquels s'inscrivent ces PME présentent une configuration différente de celles que l'on avait analysées dans le cas du système industriel local d'Oyonnax (Raveyre et Saglio, 1984). Plus endogènes au site, ils reposaient essentiellement sur des relations de coopération - concurrence entre entreprises locales du même secteur de la transformation des matières plastiques. L'étude des relations privilégiées que les PME de notre échantillon entretiennent avec d'autres entreprises, conduit à mettre en évidence un autre maillage dépassant le niveau local, avec l'articulation de deux types de réseaux.

Les réseaux les plus développés sont verticaux, avec les clients et fournisseurs nationaux/internationaux. Ils contribuent à assurer de la qualité des produits et l'efficacité technique de ces PME. En effet, les relations avec les clients sont étroites afin d'apporter une réponse adaptée aux particularités de la demande. De même, les relations avec les différents partenaires industriels, qu'ils soient fournisseurs ou soustraitants, sont souvent fortes, dans le but de parvenir à une maîtrise de l'ensemble de la chaîne opératoire. Par exemple, une PME fabriquant des machines spéciales, échange des informations avec son fournisseur concepteur de programmes (situé dans une autre région), et discute avec son sous-traitant fabricant de pièces, pour définir une machine en cours de conception. Ces pratiques, le plus souvent non formalisées dans des contrats explicites, reposent essentiellement sur des ententes interpersonnelles. La nature des échanges concerne ici des services: informations techniques et commerciales, conseils, etc. De cette façon, ces PME se donnent la possibilité d'accéder à des services diversifiés, qu'elles n'auraient pas eu les moyens d'entretenir seules ; soit de mobiliser des ressources sans alourdir leurs investissements.

2 Ces PME sont aussi parties prenantes de réseaux horizontaux, entre PME collègues locales. Nombre d'entre elles font appel à leurs homologues de proximité (de différents secteurs d'activité), pour faire face aux variations en qualité et en quantité de leur fabrication. Afin de répondre aux demandes des clients, parfois diverses et ponctuelles, il leur arrive de louer temporairement du matériel, des locaux ou de la main-d'œuvre à des PME voisines, qui elles-mêmes donnent des coups de main dans d'autres occasions. Par exemple, une PME de transformation des matières plastiques, fabriquant habituellement des produits de petite taille, loue temporairement l'entrepôt d'une entreprise voisine, pour répondre à une commande de coques de voiliers.

Ainsi, si le recours aux réseaux verticaux contribue à l'efficience technique et à la qualité des produits, celui aux réseaux horizontaux concourt à assurer la souplesse productive et la mobilité des entreprises sur le marché ; deux aspects qui touchent au cœur même du fonctionnement de ces PME. Cette articulation singulière de réseaux constituerait l'un des facteurs de performance de ces PME, simultanément enracinées dans l'espace local et intégrées à l'espace national (et pour certaines international).

\section{Flexibilité du travail et de l'emploi}

4 La gestion des ressources humaines de ces entreprises est marquée par une recherche d'adaptation constante du facteur travail, afin d'atteindre les objectifs de l'entreprise en terme de qualité, d'élasticité technique et de réactivité à la demande. En cela, elle 
s'apparente, à bien des égards, aux nouvelles formes d'organisations du travail postfordiennes, misant sur la mobilisation des compétences (Zarifian, 1999), où les salariés doivent faire preuve d'autonomie, d'initiative et d'adaptation. Or, contrairement à certains présupposés, il semblerait que ces pratiques trouvent dans la société rurale française contemporaine un terrain particulièrement favorable ${ }^{8}$.

\section{La valorisation de compétences polyvalentes et autonomes}

L'emploi dans ces PME est composé majoritairement d'ouvriers, spécialisés et qualifiés. Si le niveau moyen de formation n'est pas élevé, les salariés doivent cependant mettre en œuvre des compétences polyvalentes et autonomes. La nature des taches peut être relativement variable: suivant la quantité et la nature de la demande du client, l'organisation du travail est susceptible d'être modifiée ainsi que la répartition des salariés sur la chaîne opératoire. Une telle mobilité des salariés à l'intérieur de l'entreprise nécessite des savoirs polyvalents, chacun étant amené à occuper des postes différents, ne correspondant pas toujours à sa qualification d'origine. Ceci peut concerner des salariés de divers niveaux : ainsi un technicien accomplira une tâche de manœuvre durant une semaine, parce qu'il y a un coup de bourre. On remarquera que les compétences polyvalentes et autonomes reposent sur une culture technique de la même famille que celle du modèle pré-fordien, - lié à l'agriculture et à l'artisanat -, qui, de fait, se trouverait particulièrement adaptée aux nouveaux modes de produire postfordiens contemporains.

Le niveau de qualification et de salaire dans ces entreprises, n'est pas plus bas qu'ailleurs. Pour les dirigeants rencontrés, la recherche du moindre coût de la maind'œuvre passe le plus souvent après la qualité de celle-ci, - ce qui marque la différence avec le modèle d'implantation industrielle fondé sur la recherche de main-d'œuvre à bas prix dans les campagnes.

\section{Mobilité du personnel et variation du temps de travail}

Le recrutement des employés et ouvriers a une forte dominante locale, mais ce n'est pas au sens étroit du terme. Les salariés habitent dans un périmètre moyen de cinquante kilomètres des entreprises. On peut voir là un effet de l'évolution des modes de vie ruraux des dernières décennies, où la mobilité de la population s'est considérablement accrue. Les techniciens et cadres, pour leur part, viennent souvent d'autres régions. On notera que l'une des difficultés rencontrées par ces PME, particulièrement celles situées dans de très petites communes, peut être de trouver du personnel correspondant aux besoins de l'entreprise.

La gestion de la main-d'œuvre, s'appuie de façon importante sur des modes de régulation informels du temps de travail. Fréquemment, on fait des heures en plus, le soir, ou le week-end. Si le paiement en heures supplémentaires est rare, les possibilités de récupération sont souvent la règle. On peut prendre des journées de congés, ou partir à certaines heures pour des raisons personnelles. Très souvent cela se réalise de façon informelle, par des arrangements interindividuels. Par ailleurs, c'est souvent la durée des contrats de travail qui varie, avec des recours répétés, en appoint, à des contrats à durée déterminée ou à temps partiel. Ces pratiques sembleraient facilitées en zone rurale, car la population, du fait de la fréquente pluriactivité des ménages, ou de la 
possibilité de loisirs liés à la nature, s'accommoderait plus facilement de cette élasticité d'ensemble du temps de travail.

Cette organisation flexible du travail, qui sollicite accords interpersonnels et compétences des salariés, reposerait, pour partie, sur une mobilisation subjective des salariés. La représentation consensuelle de l'entreprise comme une équipe, proposée par les dirigeants, ne serait pas ici sans jouer un rôle pour susciter l'adhésion du personnel (lequel, par ailleurs, paraît peu marqué par la culture syndicale ouvrière) ${ }^{9}$.

Le mode de gestion du travail, observé dans ces PME, allie donc tradition et modernité, ententes interpersonnelles et adhésion au projet d'entreprise. En cela, ce type de gestion, de la famille des nouveaux modes de management participatifs, misant sur la flexibilité et la mobilisation des salariés, semblerait bien s'adapter au milieu rural contemporain, qui présente des caractères culturels mixtes. On peut avancer, à titre d'hypothèse, que la main-d'œuvre rurale, liant un héritage culturel - technique préfordien avec des modes de vie modernes basés sur la mobilité et la pluriactivité, se présenterait comme un potentiel de gestion flexible du travail. Ce qui aurait pu être supposé un handicap pour le développement d'une entreprise performante, apparaît, ici, comme un atout.

\section{Conclusion}

41 Les orientations prises par ces PMI au niveau commercial, technique et de la gestion de l'emploi, présentent une cohérence d'ensemble, qui confère à ces entreprises un mode de fonctionnement particulier, dont la caractéristique essentielle peut être définie comme une capacité globale d'adaptation. Celle-ci apparait comme une valorisation ingénieuse des possibilités imparties par l'état du marché et des techniques. L'étude de ces entreprises rappelle donc qu'il existe moins des standards de développement que des ajustements à chaque fois singuliers. Le mode de fonctionnement de ces entreprises porte la marque du système de valeurs de ces dirigeants d'un type particulier. Ces cadres supérieurs, remettant en cause les modèles d'entreprises et envisageant sans réticence innovations technologiques et ouvertures commerciales, auraient su trouver en milieu rural des possibilités de développement industriel que les entrepreneurs traditionnels semblent ne pas toujours percevoir. Les raisons de la performance de leur entreprise se trouveraient donc moins du côté de ressources objectives qui auraient fait défaut à d'autres, que dans l'élaboration de solutions originales, pour lesquelles leur véritable patrimoine semble surtout socioculturel.

Si cette étude de cas conduit à esquisser les contours d'une nouvelle forme de PME, celle-ci ne serait cependant pas une exclusivité du milieu rural, bien qu'elle trouverait ici un terreau favorable. En effet, des PME de types apparentés ont pu être observées ailleurs (Ardenti et Vrain, 2000 ; Raveyre, 1988). Ces PME correspondraient à un modèle cellulaire en réseaux, fondé sur la flexibilité et l'intégration dans des réseaux interentreprises dépassant l'espace local. Le monde rural ne serait donc pas plus délaissé par les nouvelles formes d'entreprenariat, que voué aux types d'industrialisation connus antérieurement. Cette étude de cas, qui ne saurait être représentative de l'état des PME rurales, permettrait surtout, en jouant un rôle de révélateur, de dégager quelques pistes quant aux possibilités actuelles de développement des PME en milieu rural. Ces possibilités, semblent moins liées à un type d'activité et à des spécificités locales qu'à des évolutions d'ordre général: un 
changement social dans une certaine catégorie d'entrepreneurs, le développement des nouvelles technologies, les changements des critères de la concurrence donnant plus de place à la qualité et à la diversité.

43 Les atouts du milieu rural, tels qu'ils se dessinent ici, sont relativement déconcertants. Le premier tiendrait aux qualités socioculturelles de la main-d'œuvre rurale, qui, liant des caractéristiques anté-fordiennes avec des modes de vie modernes, sembleraient relativement bien s'accommoder des modes de management post-fordiens. Un autre aspect doit aussi être évoqué, aspect guère considéré classiquement comme facteur d'industrialisation : l'environnement naturel. Il semble, en effet, que l'attrait que celuici exerce sur certains entrepreneurs, du fait d'une conception particulière de qualité de vie (dans et hors travail), ait joué un rôle non négligeable, lors du choix du lieu d'implantation.

Si cette étude conduit à émettre l'hypothèse que de nouvelles perspectives s'ouvrent aux petites entreprises industrielles en milieu rural, il s'agirait à présent de mener plus avant la réflexion en élargissant l'observation. Resterait notamment à s'interroger quant aux types de productions susceptibles de s'adapter ou non à cette forme d'industrialisation, à repérer les espaces ruraux les plus attractifs pour ce type d'entreprise et à mieux appréhender la dimension sociale du phénomène, du point de vue de la population des campagnes.

\section{BIBLIOGRAPHIE}

ARDENTI (R), VRAIN (P.), 1998. - Les PMI et leurs dirigeants. Stratégies de marché et stratégies internes, Dossier du Centre d'Études de l'Emploi, n 17, Paris, La Documentation française, 133 p.

BAGNASCO (A.), 1977. - Tre Italie : la problematica territoriale dello sviluppo Italiano, édition Il Mulino, Bologne, $255 \mathrm{p}$.

BeCATtini (G.) (dir.), 1987. - Mercato e forze locati : il distretto industriale, édition Il Mulino, Bologne, $193 \mathrm{p}$.

BOISSARD (P.), LETABLIER (M.-T.), 1987. - « Le camembert : normand ou normé. Deux modèles de production dans l'industrie fromagère ", dans Entreprises et produits, Cahiers du Centre d'Études de l’Emploi, n³ 30, Paris, PUF, p. 1-29.

BONTRON (J.-C.), 1991. - Les dynamiques récentes de création et de localisation des activités en milieu rural, Paris, DATAR/SEGESA, 21 p.

BUNEL (J.), SAGLIO (J.), 1979. - L'action patronale en France : du CNPF aux petits patrons, Paris, PUF, 250 p.

COURLET (C.), PECQUEUR (B.), 1988. - « Un modèle de développement industriel diffus : le Choletais », Histoires de développement, Cahiers de l'institut d'études sociales de Lyon, $\mathrm{n}^{\circ}$ 4, p. 26-31.

GuYOT (M.), 1987. - « Nouvel entrepreneur ou nouveau travailleur ? Le créateur d'entreprise », Technologie, Idéologie, Pratique, vol. 6, n 4, p. 49-74. 
INSEE, 1996. - Les créations d'entreprises du premier semestre 1994, INSEE Résultats Système productif, $\mathrm{n}^{\circ} 97-98,336 \mathrm{p}$.

JULIEN (P.-A.), MARChESNAY (M.), 1988. - La petite entreprise, Paris, Vuibert, 287 p.

KAYSER (B.) (dir.), 1993. - Naissance de nouvelles campagnes, Paris/La Tour-d'Aigues, DATAR/Édition de l'Aube, $174 \mathrm{p}$.

RAVEYRE (M.), 1988. - Jeux de Miroirs. L'aide de Saint-Gobain-Développement aux PME (Rapport pour le CNRS et le ministère de la Recherche), Lyon, GLYSI-CNRS, 250 p.

-, 1999. - De nouveaux acteurs pour le développement local : les grandes entreprises. (Rapport pour le Commissariat général au plan et la Dares), Paris, Centre d'Études de l’Emploi/GIP MI, 196 p.

RAVEYRE (M.), SAGLIO (J.), 1984. - « Les systèmes industriels localisés : éléments pour une analyse sociologique des ensembles de PME industriels ", Sociologie du travail, n 2, p. 157-176.

TROUVE (Ph.), 1989. - « La construction sociale de pratiques entreprenariales : le cas de la microrégion thiernoise ", dans HADJADJ D. (dir.), Pays de Tiers, le regard et la mémoire, Clermont-Ferrand, Institut d'études du Massif Central, $50 \mathrm{p}$.

VelTz (P.), 2002. - Des lieux et des liens, La Tour-d'Aigues, Éditions de L'Aube, 153 p.

ZARIFIAN (P.), 1999. - Objectif compétence, Paris, Liaisons, 229 p.

\section{NOTES}

1. Bien que mise en débat.

2. Ce qui peut être appréhendé comme un modèle de production domestique (Boissard et Letablier, 1987), dans lequel les entreprises s'appuient sur des traditions de fabrication et des liens locaux de production.

3. Les entreprises sont situées dans diverses régions, mais l'on ne saurait tirer des enseignements de leur répartition géographique, qui résulte essentiellement d'un effet d'échantillon.

4. Pour les seuls créateurs d'entreprise, 81 \% avaient un niveau inférieur au BAC en 1979, dans l'étude de J. Bunel et J. Saglio (1979).

5. Les cas étudiés ne sont pas des essaimages de grandes entreprises.

6. Ainsi, l'enquête SINE de l'INSEE, fait apparaître que 21,2 \% des créations d'entreprises, en 1994, se sont faites avec des associés.

7. Le développement de micro-groupes est d'ailleurs un des faits marquant de l'évolution des formes d'organisation productive au cours des deux dernières décennies.

8. Comme cela est désormais bien connu, la tendance de fond d'évolution de la société rurale contemporaine est marquée, notamment, par la croissance de la mobilité locale de la population active, une augmentation du niveau de formation, le développement de la pluriactivité des ménages agricoles (voir en particulier B. Kayser, 1993).

9. Mais il resterait à étudier plus avant dans quelle mesure et suivant quelles modalités les salariés adhèrent où subissent cette forme d'organisation. 


\section{RÉSUMÉS}

Dans le contexte actuel de reconfiguration du système productif, il semblerait que de nouvelles perspectives se dessinent pour le développement de petites entreprises industrielles en milieu rural. En effet, l'étude de cas d'une vingtaine de PMI en expansion créées au cours des deux dernières décennies dans diverses zones rurales non périurbaines françaises, conduit à dégager une forme spécifique de PMI. Celle-ci présente un profil de dirigeant original de néoentrepreneurs ruraux, des anciens cadres urbains avec des valeurs éloignées de celles des patrons de PME traditionnels proches des nouvelles formes de management. Les orientations données à leur entreprise, misent sur la qualité et la spécialisation des produits, la flexibilité du travail et l'inscription dans des réseaux ne se limitant pas à l'espace local, mais intégrant le niveau national, voire international.

In the present context in which the system of production is changing shape, it seems that new prospects are emerging for the development of small industrial businesses in rural areas. Indeed, the case study of some twenty expanding SMEs set up in the last two decades in various rural and not peri-urban areas of France has led to the identification of a specific form of SME. This shows an original management profile for rural neo-entrepreneurs, former urban executives with values that are a long way from those of traditional SME managers close to the new forms of management. The approach to their enterprise is based on quality and product specialisation, flexible working and involvement in networks which are not limited to the local area, but extend to the national and even national level.

\section{INDEX}

Mots-clés : patronat, mode de développement, petite entreprise industrielle, espace rural

Keywords : development model, small industrial businesses, rural area

Index géographique : France

\section{AUTEUR}

\section{MARIE RAVEYRE}

IRES (Institut de Recherches Economiques et Sociales), 16 Bd du Mont d'Est, 93192 Noisy-LeGrand cedex, France,

Marie.Raveyre@ires-fr.org 